\title{
PULMONARY ARTERIAL HYPERTENSION IN SYSTEMIC LUPUS ERYTHEMATOUS
}

Pablo Vinicius da Fonseca1, ${ }^{1, *}$, Jean Michell Correia Monteiro ${ }^{1}$, Karina Pucha Aguinsaca ${ }^{1}$, Marina Barguil Macedo ${ }^{1}$, Samuel Katsuyuki Shinjo ${ }^{1}$, Diogo Souza Domiciano ${ }^{1}$

1.Universidade de São Paulo, São Paulo (SP), Brazil.

${ }^{*}$ Corresponding author: pabloviniciusfonseca@gmail.com

\section{BACKGROUND}

Pulmonary hypertension $(\mathrm{PH})$ is an entity of high morbidity and mortality, with multiple facets and, therefore, several treatments. It can be primary or secondary and it is not uncommon complication in collagenosis and does not always have systemic activity.

\section{CASE REPORT}

Female patient, 33 years old, with systemic lupus erythematosus since 2011, early arthritis. In 2015, she reported intense dyspnea and the investigation showed $\mathrm{PH}$ in addition to hemolysis, thrombocytopenia and pleuritis. Methylprednisolone and cyclophosphamide (monthly, for eight months) were started on pulse therapy doses. Bosentan and sildenafil, followed by azathioprine and progressive reduction of the prednisone. After 36 months, there was a new progressive and refractory worsening of dyspnea, without other signs of activity and, so, did not received immunosuppression adjustment. She was admitted in April 2021 for disabling dyspnea. During hospitalization, she needs high oxygen flows for therapy (face mask with $15 \mathrm{~L} / \mathrm{min}$ ); however, she still had hypoxemia with a maximum of $80 \%$ peripheral saturation, central cyanosis, but her general condition was preserved (she smiling, eating without assistance, no change in her consciousness) and "without" systemic activity. She performed right cardiac catheterization (mean pulmonary arterial pressure $58 \mathrm{mmHg}$, pulmonary vascular resistance 13.6 Wood units, pulmonary arterial wedge pressure $9 \mathrm{mmHg}$ ); echocardiography with normal left ventricle and right ventricular overload, in addition to signs suggestive of right-to-left shunt; a cardiac nuclear magnetic resonance just with change in right ventricle; CT angiography and chest scintigraphy without signs of pulmonary embolism, with radiopharmaceutical in systemic circulation. It was triple therapy for $\mathrm{PH}$ with bosentan, sildenafil and iloprost and, after exclusion of infection, pulse therapy with methylprednisolone. The patient showed significant improvement, so she was treated with cyclophosphamide (monthly planning for 6 months) and after 20 days, she had peripheral saturation greater than $95 \%$ with no additional oxygen.

\section{CONCLUSION}

Pulmonary hypertension is a serious complication of collagenosis, the right cardiac catheterization is sine qua non for correct treatment and the main mechanisms are vasculopathy and vasculitis. When we have vasculitis, immunosuppression is part of the treatment and disease activity cannot be underestimated, even in patients with few signs or low index of activity. There is no consensus on the optimal treatment. The specific therapy against $\mathrm{PH}$ is recommended, furthermore, in contrast to the other severe manifestations, the picture if low systemic activity is often observed.

\section{KEYWORDS}

Systemic lupus erythematosus, Lower activity, Pulmonary hypertension. 